\title{
X-linked intellectual disability, Abidi type
}

INSERM

\section{Source}

INSERM. (1999). Orphanet: an online rare disease and orphan drug data base. $\underline{X \text {-linked }}$ intellectual disability, Abidi type. ORPHA:85273

X-linked intellectual disability, Abidi type is characterized by X-linked intellectual deficit and mild variable manifestations, including short stature, small head circumference, sloping forehead, hearing loss, abnormally shaped ears, and small testes. It has been described in eight affected males from three generations. 